\title{
VALOR DE UNA INCÓGNITA: EL TRABAJO DEL PSICÓLOGO EN INSTITUCIÓNES DE SALUD
}

\section{Value of an unknown factor: the work of a psychologist in health institutions}

\begin{abstract}
(20)
Julio C. Guillén*

\section{Resumen}

La presente comunicación trata del trabajo del psicólogo en las instituciones de salud mental, en particular en el centro médico psicológico (CMP), y también en otras instituciones de enseñanza como la universidad. En ambos casos, plantearemos la cuestión del lugar del psicólogo en el seno de la institución y al mismo tiempo de sus relaciones con los otros profesionales, los pacientes o los estudiantes. Surge la pregunta: ¿es posible caracterizar la posición del psicólogo en institución de un punto de vista formal, sin fijarla al mismo tiempo y en el mismo movimiento?

Aún admitiendo que poseemos un cierto saber, como testimonia un diploma, ¿se trata de hacer valer este saber, o más bien de posicionarnos como "representantes" (faire-valoir) de un saber "otro"?

La experiencia clínica muestra que el saber universitario encuentra sus límites en la particularidad de cada caso. De este modo, volvemos a la pregunta anterior transformada:

La articulación sui-generis que propone el psicoanálisis entre teoría y práctica, constituirá nuestra referencia para tratar de responder a esta pregunta. Presentaremos la experiencia de creación de un "grupo de reflexión clínica" en CMP, espacio de encuentro (y desencuentro) de distintos profesionales que interroga particularmente el lugar del psicólogo.
\end{abstract}

Palabras clave: Trabajo del psicólogo, instituciones de salud.

\begin{abstract}
The present communication is related to psychologist's work in mental health institutions, particularly in the Medical Psychology Center as well as other teaching institutions as the university. In both cases, we will explain the psychologist's position within the institution and at the same time their relations with other professionals, patients or students. The question arises: Is it possible to characterize the position of a physichologist in the institution from a formal point of view, without stating at the same time and at the same movement? Even admitting that we have a certain knowledge, as it testifies a diploma, it is about asserting this knowledge, or positioning as "representatives" (faire-valoir) of a knowledge "other"?

The clinical experience shows that university knowledge finds their limits in the particularity of each case. In such aspect, we come back to the modified previous question:

The sui-generis articulation suggests that psychoanalysis among theory and practice will constitute our reference to answer this question.

We will introduce the creation experience of a "clinical reflection group" in The Medical Psychology Center, finding and discarding of different professionals which interrogates particularly the psychologist's position.
\end{abstract}

Key words: work of the psychologist, health institutions.

* Físico y Psicólogo. Docente investigador. Laboratoire SHS-CEC - Unité de Recherche en Psychologie OCeS (Organization Clinique et Sujet) Université Catholiqye de Lille- Facultad Libre des Lettres et sciences Humanines, julio.guillen@icl-lille.fr 


\section{INTRODUCCIÓN}

Desde hace diez años mi actividad académica y profesional se desarrolla en Francia.

La "escuela francesa" es una referencia presente en general en los recorridos académicos en Argentina. $\mathrm{Al}$ menos esta ha sido mi experiencia a lo largo de mis estudios universitarios y lo que he podido escuchar de la mayoría de los colegas argentinos con quienes me he encontrado a lo largo de estos años de ejercicio profesional y académico. En el campo del ejercicio profesional en instituciones y en relación con los servicios hospitalarios en particular, si bien la enseñanza explícita de su funcionamiento no es sistemáticamente desarrollada, en las pasantías primero y luego en las residencias nos confrontamos con la evidente influencia francesa en la organización de la práctica. Aún así, en estos años de actividad profesional, he podido constatar que las diferencias entre ambos lado del Atlántico no son pocas. Quisiera entonces, a través de este trabajo, dejar filtrarse las resonancias de las particularidades que distinguen Francia de la Argentina, en cuanto al contexto de la problemática que presentaré a continuación.

La influencia de la clínica y de la teoría francesa en el campo "psi" en Argentina agudiza probablemente el contraste entre los dos países en la enseñanza y la práctica de la psicología en cuanto a ciertos detalles. En particular en cuanto a la presencia y al rol del psicoanálisis -especialmente del psicoanálisis lacaniano-tanto en las instituciones hospitalarias como en la universidad.

Si bien puede hablarse en cierto modo del campo de la psicología como disciplina en general, como es sabido, este campo está muy lejos de constituir una totalidad coherente y homogénea. Me referiré entonces específicamente a los desarrollos psicoanalíticos que constituyen el fundamento de mi práctica y de mi actividad académica y de investigación. Hablaré del "rol del psicólogo" puesto que es como "psicólogos" que somos llamados a integrar las instituciones en universitarias y hospitalarias. No dejamos entonces de hacer frente a esta paradoja: el rol del psicólogo no es algo generalizable. Y no solamente porque son múltiples las tareas que puede desempeñar, sino porque su práctica y su saber se constituyen en un campo que no puede no llevar la marca de la falta. Falta que, si bien representa una falla, es fundadora de lo humano en su dependencia del significante. Psiquis y Soma son indisociables, pero no armonizables. Están "mal cosidos", y lo estarán siempre, no sólo en los casos patológicos. En efecto, el psicoanálisis introduce el montaje pulsional, lo que permite explicar cómo los agujeros del significante se superponen imperfectamente a los bordes del cuerpo -esto en el mejor de los casos- y el surgimiento de la dimensión "agujereada" del inconciente.

Aquí puede situarse la impostura de la vocación cientificista de una psicología que quisiera presentarse como una ciencia en su carácter de conocimiento universal y como técnica de intervención. No es en el uso supuestamente deformado de conceptos de otras disciplinas que se encuentra esta impostura, como han podido afirmarlo Brickmont y Sockall (Sokal \& Bricmont, 1997), sino en la voluntad de forzar la inclusión de la psicología en las ciencias naturales. Sin embargo, distinguirse de la ciencia como Weltanschauung no implica de ningún modo una renuncia a la precisión o al rigor en la investigación y menos aún que no sea necesario confrontar las hipótesis con la experiencia.

El psicoanálisis viene justamente a señalar este fenómeno fundamental de toda teorización de los procesos "anímicos", como se los denominaba antiguamente, y de toda práctica que tenga en cuenta el sufrimiento psíquico, que no se trata de una carencia de medios o una limitación en los conceptos, sino de una falla constitutiva: "El ejemplo de la ciencia física puede sin embargo mostrarles que los más grandiosos éxitos no implican que sepamos a donde vamos." (Lacan, 1958, p. 615).

Es en este sentido que podemos plantear la cuestión del "lugar del psicólogo", en términos de posición, ligada tanto al valor ético como al valor de una incógnita. En el primer caso, se trata del rigor para sostenerse en una relación específica -lo que en absoluto significa rigidez-, y en el segundo caso se trata del rigor formal en el marco de una dinámica determinada. No debe confundirse aquí posición con postura, la que siempre está en relación con la forma y por lo tanto con una Gestalt imaginaria (utilizo el 
pleonasmo intencionalmente) - que se reduce en última instancia a un mero semblante.

\section{EL PSICÓLOGO COMO EXPERTO}

El psicólogo, si es convocado a un lugar de experto en la Universidad o en el Hospital, lo es desde otro lugar que lo posiciona como poseedor de un saber o de una técnica, lo que nos envía directamente a situarlo ya sea en el discurso del amo como "otro" o en el discurso universitario como "agente" (Lacan, J. 196970).

El ejemplo típico en la universidad es el del profesor que imparte una enseñanza preestablecida, verificada, y que espera que sea aprendida por el alumno para luego ser evaluada. En el caso del hospital el ejemplo paradigmático es el de la evaluación psicológica. El psicólogo, especialista en la evaluación del funcionamiento psíquico y sus patologías, sería quien, aplicando las técnicas adecuadas, puede pronunciarse sobre la normalidad o el grado de normalidad del paciente.

Aún más, hoy en día no se trata ni siquiera del funcionamiento psíquico con toda la complejidad que progresivamente pudo incorporar tanto del psicoanálisis como de la psiquiatría clásica: categorías psicopatológicas, descripciones clínicas, dinámica del inconciente, mecanismos de defensa, desarrollo del yo, etc. Se trata del "estado cognitivo y emocional" del paciente y de su capacidad de adaptación. La consecuencia directa es que a partir del diagnóstico se aplicarán las técnicas adecuadas para resolver aquello que se presenta como problema. No decimos que el alivio del o de los síntomas no sea benéfico en ciertos casos (que como todos ellos deben ser analizados en su particularidad). Sino que lo que se elude es la formulación de la pregunta. Y con esto, evidentemente, la cuestión del sujeto. Volveremos más adelante sobre esto.

Por lo tanto, hablamos hoy en día de 'individuos', representantes de una clase genérica, definidos en intensión por ciertas variables que los caracterizan y por su medición en términos cuantitativos que permiten saber cuál es el grado de alejamiento del ideal de la normalidad. Con esto se crean nuevos conjuntos que sirven para englobar desviaciones similares.
Reproduzco aquí, a título de ilustración, la demanda de uno de los psiquiatras del servicio dirigida al psicólogo durante una reunión de síntesis:

Vi a la Sra X en primera entrevista ayer, 37 años ... dice que siente un rechazo por el trabajo, que todo le resulta difícil, que no llega a tiempo o no comprende bastante rápido ... no me da la impresión de una patología psiquiátrica ... su médico de cabecera la envía por una depresión pero yo no encuentro signo alguno, no hay tristeza de fondo, ni autodegradación, bradipsiquia, ideas de muerte .... Puede ser un problema de capacidad para el trabajo... te la mandé para hacer un QI.

En otras oportunidades los pacientes son descritos como "un manipulador" o "un provocador".

Toda dimensión subjetiva se cierra de este modo para ambos, para el paciente la pregunta de ¿qué digo con mi decir, de qué me quejo? Y para el psicólogo ¿qué quiere de mí? No sólo no hay pregunta abierta por la situación de la persona que viene a consultar $-\mathrm{y}$ menos aún por la necesidad misma de construir la pregunta -, sino que los síntomas se reducen a signos (bradipsiquia, humor triste, ideas de autolisis), y el llamado a intervenir al psicólogo con una mirada de experto.

Insistimos, no se trata del valor en sí mismos de los instrumentos como las escalas de QI, los tests proyectivos o de personalidad, lo que podría discutirse largamente. Se trata de la posición del psicólogo que rápidamente puede quedar prisionero de su captura imaginaria por parte de la institución.

No es cuestión tampoco de negar todo saber, porque esto nos llevaría a una especie de conocimiento intuitivo, individual, casi místico e inefable. Todo el esfuerzo de Freud en diálogo constante con la medicina, la biología, las ciencias físicas, demuestran lo contrario. Pensamos que el espíritu del "Proyecto" (Freud, 1895), de hacer entrar la psicología en el dominio de las Ciencias Naturales, aunque sólo se trata de un "prototexto", acompañó a Freud a lo largo de toda su obra. Al mismo tiempo, cabe señalar que sus límites mismos se hicieron manifiestos rápidamente a través de la práctica y de su necesidad de incorporar a la reflexión 
la literatura (Freud, 1919), la mitología (Freud, 1922) o los estudios sociales (Freud, 1913). Lo mismo puede afirmarse en el caso de Lacan. Si de un lado tenemos la lingüística, la lógica y la topología, del otro tenemos la literatura (Lacan, 1975-76), el arte (Lacan, 196364) y la filosofía (Lacan, 1960-61). Esto responde a la múltiple determinación de lo humano ante la cual no retroceden.

Actualmente, esta multiplicidad no armonizable es lo que se trata de evitar cuidadosamente en cada caso para "formatearlo" con la "máquina del saber científico". Las posiciones son aquí claras: el psicólogo es una mirada experta reducida a sus instrumentos de observación y medición, el paciente el objeto de estudio. Casi podrían obviar su presencia respectiva (lo que en un extremo casi caricatural constituyen los test autoadministrados y evaluados informáticamente, o las técnicas de auto-ayuda).

No hay lugar para el mal entendido, la duda. Se trata de reducirlos y así ganar tiempo. Pero, ¿tiempo para qué y para quién? Evidentemente para la institución que así no tendrá que "soportar" largas curas u hospitalizaciones o largas psicoterapias que son pesadas para el presupuesto y no alargan la "fila activa" (número de pacientes a cargo del profesional). Es tiempo también para el paciente, para que retome su vida lo más rápidamente posible. ¿Pero qué vida, la anterior con sus síntomas y su padecer, era menos suya?

Este concepto de lo extranjero coincide con la caracterización del sufrimiento como una enfermedad, algo que irrumpe en la vida. Recordemos la antigua concepción de la enfermedad como un cuerpo extraño que se instala en el organismo o en el psiquismo y produce una anomalía, una ruptura de la armonía, sin olvidar aquella que la presenta como algo que el mismo cuerpo lo produce, alimenta, como un tumor. En los dos casos se trata de aquello de lo que hay que liberarse, que no me pertenece.

Freud inauguró una ruptura con este tipo de modelo. En un primer momento lo reprimido fue presentado en este sentido, como un complejo inaccesible al paciente que produce los síntomas de los que se queja y que debe, a través de un procedimiento particular, ser restituido a la conciencia y de este modo disuelto. Pronto esta primera visión mostró sus límites en la práctica y también en el dominio teórico como consecuencia de la exploración de los procesos "normales" -sueño, lapsus, chiste-que responden a la misma dinámica que el síntoma. De este modo, el núcleo extranjero ya no era patógeno en sí sino que constituía lo más íntimo de la persona y la verdad del sujeto anclada en la contingencia de su propia historia.

Lacan, por su parte desarrollará esta perspectiva a lo largo de toda su enseñanza, ya sea a través de la introducción del sujeto dividido por el lenguaje y su relación al Otro o más adelante los desarrollos sobre el objeto $a$ (Lacan, 1962-63), siempre teniendo en cuenta que en su caso los conceptos no se sustituyen unos a otros.

Se plantea aquí la cuestión central del valor del síntoma. Lejos de una investigación en la que el analista se extrae como observador de una situación patológica, visto que el síntoma, en el marco analítico, se construye en transferencia, la pregunta acerca de su valor en la constelación subjetiva no puede no concernirlo.

Del mismo modo, la posición de quien intenta transmitir el psicoanálisis en la Universidad, no puede coincidir con la del poseedor de un saber, haciendo del estudiante una "máquina de aprender" las teorías en boga. Recordemos que toda máquina trabaja con una pérdida, una producción de entropía que no es algo a eliminar sino justamente el punto de articulación con lo real en la experiencia (Lacan, 1969-70).

Si bien en este caso la demanda es del estudiante que se inscribe en la carrera, la falla del saber deberá poder situarse del lado de quien se propone para la transmisión, al menos como horizonte de todo conocimiento sistemático. Y es justamente por ello que deberá poder hacer un "tour" ("vuelta" y "truco" en francés) a la demanda original para retornar al estudiante de manera invertida. Reenviarlo al límite de todo conocimiento es una forma de hacer presente la verdad. Esto no quiere decir que se parta de la ignorancia absoluta. Se parte de conceptos, de nociones, de recorridos históricos, de casos clínicos, pero en última instancia para des-construirlos, para señalar en la vacilación del corpus scientiae la dirección 
de la verdad a la que indefectiblemente lo confrontará la práctica clínica.

\section{EL GRUPO DE REFLEXIÓN CLÍNICA}

Después de haber trabajado seis años en el EPSM (Establecimiento Público de Salud Mental) Lille Métropole, pedí incorporarme al EPSM Lille Agglomération que se ocupa de otro sector psiquiátrico de la región. Luego de mi llegada, en varias oportunidades, el Jefe de Servicio a quien ya conocía de mi actividad institucional precedente, expresó la idea de que era necesario un tiempo de reunión para que los miembros del equipo que lo desearan pudieran profundizar la reflexión sobre ciertos pacientes. En particular, sobre aquellos que presentan situaciones problemáticas, que suscitan dudas diagnósticas o simplemente para que los diferentes miembros del equipo puedieran escucharse en relación a una persona de la que se ocupan cada uno por separado.

Aquí se plantean varias preguntas: entre otras, ¿Por qué se me dirige a mí esta demanda? ¿Por qué en este momento? ¿Cuál es el objetivo? ¿Existe un malestar particular en la institución? ¿Hay una historia de grupos de reflexión previos?

Todo esto debe asociarse a mi llegada reciente, aproximadamente 6 meses antes, y al hecho de que mi práctica tiene como marco de referencia al psicoanálisis.

Decidí entonces hacer una proposición al equipo para constituir un grupo de reflexión clínica, explicando cuál había sido la demanda en principio y al mismo tiempo mi posición en cuanto a la importancia de un espacio de palabra en relación a la práctica. En una institución en que se fragmenta progresivamente la intervención de cada miembro, un tiempo consagrado a la puesta en común y al cuestionamiento, puede sobre todo servir para abordar la cuestión del sentido de las diversas intervenciones. Por otro lado, es fundamental que quien dirige la institución haya sido sensible a esta necesidad y que haya propuesto que el tiempo del grupo se incluya en el tiempo de trabajo del equipo y no como una "actividad extra".
No me detendré en los detalles de la organización. Basta decir que fijamos el cuadro y la forma de las reuniones: tres semanas consecutivas por mes, una hora y media por semana, en principio sobre un mismo caso, que sería presentado por uno o varios miembros del equipo y, aun cuando el grupo sería abierto, se solicitó que quienes participaran se comprometieran durante al menos un mes, es decir a lo largo de la presentación de un caso. En cuanto al funcionamiento, obviamente pueden hacerse paralelos y confrontaciones con los grupos de trabajo o la psicoterapia institucional sobre los que no nos detendremos en este trabajo.

En el momento de discutir estas cuestiones, surgieron reticencias y dudas debidas, por ejemplo, a los horarios de intervención de los enfermeros en los talleres terapéuticos, a la agenda abarrotada de los médicos o a las vacaciones desfasadas del personal. No dimos una respuesta y sugerimos que la particularidad de nuestra institución nos mostraría el grado de flexibilidad a adoptar.

Al mismo tiempo, si bien todos coincidieron en la importancia y la necesidad de crear este espacio, no todos se comprometieron con él - al menos con su presencia.

Todavía más importante, ahora en el sentido de la resistencia, es que, aunque el objetivo explícito había sido enunciado, la demanda provenía del Jefe de Servicio y por lo tanto no podía desligarse de una sospecha de voluntad de control de la que yo mismo participaría.

Las preguntas siguientes se focalizaron sobre la modalidad concreta de las presentaciones. La mayoría coincidió en que era un ejercicio al que no estaban acostumbrados y que además obligaba de un cierto modo al presentador a dar cuenta de su práctica. ¿Había que partir de una anamnesis? ¿Quién se encargaría? ¿Se trataría de una reflexión conceptual a partir del psicoanálisis? ¿El médico referente del paciente debería estar presente? ¿Se haría un escrito para dejar en el expediente del paciente? Esta última pregunta, que ocupó un largo tiempo de discusión, nos deja entrever la interrogación sobre la utilidad del grupo y la transparencia de su funcionamiento. Es decir, si el grupo tiene como objetivo colaborar con el tratamiento 
del paciente, sus "resultados" deberían poder ser aprovechados por los colegas que no hubieran podido asistir para mejorar su trabajo. Expresé entonces que no era el objetivo de las reuniones llenar los «baches» de los expedientes y que había quizás otra forma de transmitir lo que habría dejado como huella el trabajo en el grupo diferente del expediente.

Pudo hablarse de estas cuestiones, en particular de la forma de abordar el caso, y se insistió sobre el hecho de que las características de la presentación quedarían a cargo del expositor y de su forma particular de concebir el caso y, sobre todo, que seguiríamos las ocurrencias espontáneas de los participantes. Una incertidumbre que no fue, ni es, fácil de aceptar.

Termino aquí esta brevísima presentación del grupo, puesto que el cuadro de esta comunicación -como todo cuadro- hace del corte una necesidad.

Sólo decir que el grupo continúa funcionando por segundo año consecutivo, con un número de participantes variable y que hemos incluido en la reflexión, en algún momento, una problemática general -como el aislamiento y la contención- y no un caso, pero siempre en relación a la práctica. Efectivamente, no puede decirse que el grupo haya entrado en una especie de funcionamiento bien aceitado y fijo. Siempre existe la incerteza de su continuación, la pregunta sobre su utilidad, el deseo o no de intervenir, las presencias o ausencias sistemáticas. Pero creemos que es justamente esto lo que permite que no se reduzca simplemente a un engranaje dentro de un funcionamiento prefijado y permita la manifestación subjetiva de los miembros del equipo.

Propongo volver ahora sobre la cuestión del valor del psicólogo con los elementos que hemos presentado.

\section{EL PSICÓlOGO COMO VALOR DE UNA INCÓGNITA}

La cuestión del "valor" está necesariamente ligada al registro simbólico en el sentido de una estructura donde los elementos tienen un estatuto diferencial y se definen por sus relaciones recíprocas. Por lo tanto la pregunta del lugar del psicólogo como valor en la institución sólo podrá responderse desde las coordenadas que permiten situarlo. Más precisamente a partir de su posición relativa y de los efectos que siguen a sus intervenciones. Por esto es imprescindible hablar de una dinámica en la que él se inserta, y más aún de la que es efecto. Es decir que no basta conocer el organigrama de la institución, se trata del modo de funcionamiento y es a partir de la dinámica subyacente que la estructura puede definirse, lo que distingue la visión dinámica psicoanalítica de la teoría sistémica.

Es en este sentido que Lacan intenta formalizar la estructura subjetiva valiéndose de figuras topológicas como la cinta de Möebius, el toro, o la botella de Klein a la que se agregan ciertas mabinobras. No es cuestión de métrica, de escalas y de distancia, sino de propiedades topológicas y para su determinación, de operaciones sobre los objetos - torsiones, cortes (Lacan, 1961-62).

Volviendo a la dimensión simbólica y tomando como ejemplo el juego de ajedrez que Freud utilizara para hablar de la sesión, el rey no vale por su substancia ni por su forma, puede ser reemplazado por un botón, siempre que podamos distinguirlo de las otras piezas. En el bridge, ejemplo utilizado por Lacan, el muerto no deja de jugar por ausentarse de la mesa, es por su mano que continúa interviniendo (Lacan, 1958).

De aquí la importancia para determinar un valor a partir del juego y de su dinámica. Pero, como en el grupo del que hablamos anteriormente, la dinámica no es un dato sino que debe descubrirse, y por lo tanto la posición del psicólogo variará según sus leyes de un modo determinado.

Es en este sentido decimos que la posición del psicólogo es una incógnita, del mismo modo que en una ecuación dinámica la posición es una incógnita. Tendrá un valor a despejar a partir de las condiciones particulares y, "del otro lado", la subjetividad en juego estará asociada necesariamente a una emergencia. Este "otro lado" está entre comillas para indicar la artificialidad de toda separación entre el costado del psicólogo y el del paciente, y de aquí la necesidad de recurrir a figuras topológicas como la banda de Möebius o la botella de Klein para construir una representación. Se puede pasar del "otro lado" sin haber atravesado un borde. El sujeto en juego, tanto en el 
tratamiento de un paciente, como en el grupo del que hablamos -pero también en el caso de la enseñanza universitaria- no pre-existe a la puesta en marcha del dispositivo y, por lo tanto, no se trata de intersubjetividad en términos de relaciones "entre sujetos".

El hecho de afirmar que existe una dinámica que determina las posiciones, no significa que haya variables para medir e introducir en una ecuación, sino que el estudio cualitativo de las propiedades dinámicas de ciertos sistemas complejos y de la lógica dialógica (Rahman et al., 1997) en particular permite continuar en la vía de Freud y Lacan a formular las preguntas en nuestro campo de manera formal en términos de "ficción explicativa". Como ya lo dijimos, el modelo tópico-dinámico- económico freudiano, o la introducción de los matemas y grafos lacanianos son pasos en esta dirección.

Lo que está aquí en juego es una distinción entre diferentes lugares para el psicólogo. Por un lado está quien se propone trabajar partiendo de la generalización de la demanda y dirigiéndose al paciente como individuo representante de una clase reducido a su síntoma: fóbico, psicosomático, etc. Por otro lado aquel que teniendo en cuenta la particularidad de la persona explora las circunstancias de su sufrimiento en posición de adelantado -como los que llegaban a Américaalguien que ha hecho la exploración y sirve de guía. Finalmente quien se sitúa de modo de conducir la cura -lo que no coincide en modo alguno con la dirección del paciente- de tal manera de ir al encuentro del sujeto en su singularidad y por lo tanto de su falla - la que no puede no evocar la nuestra. Digo singularidad en el sentido del punto de ruptura en que las leyes, las leyes dinámicas, muestran su límite y que conducen a una respuesta ética a partir del acto. Es sólo aquí que el analista como Otro ocupará una posición bien determinada y a partir de ese instante podrán leerse sus posiciones precedentes.

\section{Dos comentarios para terminar.}

En primer lugar, el hecho de ligar de manera necesaria, la posición del psicólogo a una dinámica, hace que la dimensión temporal sea fundamental. Como en el ejemplo que dimos del grupo, el tiempo que pasa no es una variable externa, sino que habrá de leerse a partir de los efectos de la puesta en marcha y de la evolución del grupo mismo. Esto es ir en contra de los tiempos que regulan normalmente la institución, calendarios, duración de entrevistas, duración de reuniones. El hecho por ejemplo de fijar una duración para el comentario de un caso no es sino el punto de partida para poner en cuestión esta regla misma. Cabe aclarar, sin embargo, que existen diferentes modos de hacerlo, y es aquí que se jugará la constitución particular del grupo de reflexión y una posibilidad para la emergencia subjetiva de los participantes. De hecho, hubo casos que ocuparon las reuniones durante tres meses. Otros que sólo ocuparon una reunión.

En una sociedad que evalúa la eficacia en horas, minutos y segundos de trabajo para una tarea, instalar como incierto el tiempo de producción de un efecto en el marco de una dinámica, significa elevar la cuestión del sujeto más allá de las capturas imaginarias y, al mismo tiempo, no poder responder a la pregunta por la posición del psicólogo más que desde este horizonte incierto de la emergencia subjetiva.

El segundo comentario retoma lo que dijimos sobre el grupo respecto al escrito para incluir en el expediente del paciente.

Algo del secreto y de la opacidad no dejó de llamarnos la atención en este planteo. ¿Cómo dejar un registro de lo que pasa en las reuniones? ¿No terminará siendo un espacio para algunos, del que los otros estarían excluidos?

La opacidad es solidaria de la posibilidad de emergencia subjetiva, cuando reconocemos en ella no la voluntad de mistificar, de tornar ilegible o inabordable un problema, sino un hecho de estructura. Como lo dijimos, la opacidad, lo turbio, indica que la «maquinaria» de saber de la que es cuestión toca lo real, la entropía y el goce (Lacan op.cit) y es en ese punto singular en que se alcanza el límite de la palabra que el sujeto, si la dinámica en juego lo permite, es llamado a responder.

En este mismo momento el psicólogo, más allá del saber que atestan sus diplomas, sus cursos, o su experiencia profesional, quedará expuesto como quien 
pone en primer plano su ausencia. Así podrá introducirse un valor para la $x$ que él ocupa -como incógnita y no como misterio -, al ser convocado como aquello que debió haber sido frente al paciente, al estudiante, al grupo; el «faire-valoir», el representante -aunque la traducción sea inadecuada- de un saber «otro» que toca, ya no el conocimiento, sino la verdad del sujeto.

\section{REFERENCIAS}

Freud, S. (1895). Proyecto de Psicología. En Obras completas Tomo I. Madrid: Amorrortu (1978).

Freud, S. (1913). Tótem y tabú. En Obras Completas Tomo XIII. Buenos Aires/Madrid: Amorrortu (1980).

Freud, S. (1919). Lo ominoso. En Obras completas Tomo XVII. Buenos Aires/Madrid: Amorrortu (1979).

Freud, S. (1922). La cabeza de Medusa. En Obras completas Tomo XVIII. Buenos Aires/Madrid: Amorrortu (1979).
Lacan, J. (1958). La direction de la cure et les principes de son pouvoir. En Autres Ecrits. Paris: du Seuil (1991).

Lacan, J. (1960-61). Le Séminaire. Livre VIII Le transfert. Paris: du Seuil (2004).

Lacan, J. (1962-63). Le Séminaire. Livre X L'angoisse. Paris: du Seuil (2001).

Lacan, J. (1963-64). Le Séminaire. Livre XI. Les quatre concepts fondamentaux de la psychanalyse. Paris: du Seuil (1973).

Lacan, J. (1969-70). Le Séminaire. Livre XVII. L'envers de la Psychanalyse. Paris: du Seuil (1998).

Lacan, J. (1975-76). Le Séminaire. Livre XXIII. Le Sinthome (1975-76). Paris: du Seuil (2005).

Lacan, J. (1961-62). L'identification. Séminaire 196162. Inédit.

Rahman S., Fishmann M., Rückert H. (1997). On Dialogues and Ontology. The Dialogical Approach to Free Logic. Logique et Analyse, 160 : 357-374.

Sokal, A., \& Bricmont, J. (1997). Impostures intellecuelles. Paris: Odile Jacob.

Fecha de recepción: 21 de abril, 2011

Fecha de aceptación: 16 de junio, 2011 